\title{
Impacto del riesgo de interés sobre las acciones del sector bancario español ${ }^{*}$
}

\section{Impact of the interest rate risk on the Spanish banking industry shares}

\author{
Laura Ballester**. Universidad de Castilla-La Mancha \\ Román Ferrer. Universidad de Valencia \\ Cristóbal González. Universidad de Valencia
}

RESUMEN Este trabajo examina la exposición del sector bancario español al riesgo de interés en el ámbito de la metodología GARCH, prestando atención no sólo al impacto de los cambios en el nivel de los tipos de interés sino también al efecto de su volatilidad sobre la distribución de los rendimientos de las acciones bancarias. Los resultados obtenidos muestran que tanto las variaciones como la volatilidad de los tipos de interés tienen un impacto negativo y significativo sobre el rendimiento de las acciones de las entidades financieras, existiendo una relación directa entre el tamaño de las entidades y su grado de sensibilidad ante los movimientos y volatilidad de los tipos de interés.

PALABRAS CLAVE Riesgo de interés; Acciones; Bancos; Volatilidad.

\begin{abstract}
This paper examines the exposure of the Spanish banking sector to interest rate risk. With that aim, a univariate GARCH model which takes into account not only the effect of the interest rate changes but also the effect of their volatility on the distribution of the Spanish bank stock returns is used. The results show that both changes and volatility of interest rates have a negative and significant impact on the stocks returns of the Spanish banking industry. Moreover, there seems to be a direct relationship between the size of banking firms and their degree of interest rate sensitivity.
\end{abstract}

KEYWORDS Interest Rate Risk; Banking firms; Stocks; Volatility.

\section{INTRODUCCIÓN}

El riesgo asociado a las variaciones de los tipos de interés constituye una de las principales formas de riesgo que afectan a las entidades financieras y tiene su origen en la peculiar naturaleza del negocio bancario. Este riesgo es inherente al tradicional papel de las entidades de crédito como intermediarios financieros que realizan una función de transformación de plazos consistente en financiar préstamos y créditos a largo plazo mediante depósitos a la vista y a corto plazo. En el entorno bancario, el riesgo de interés puede ser definido como

\footnotetext{
* Agradecimientos: Los autores desean agradecer los comentarios y sugerencias recibidos de Sonia Benito (UNED), Jorge Martínez (Banco de España), Begoña Font (Universidad de Valencia) y de los dos evaluadores anónimos de la Revista Española de Financiación y Contabilidad, así como la cesión por parte de J. Emilio Farinós (Universidad de Valencia) de su base de datos de cotizaciones bursátiles. Finalmente, agradecen la financiación recibida del Ministerio de Educación y Ciencia y de los Fondos FEDER, proyecto SEJ2005-08931-C02-02/ECON..

** Dirección para correspondencia: R. Ferrer. Departamento de Economía Financiera y Actuarial, Facultad de Economía, Avda. dels Tarongers s/n, 46022, Valencia, España. Tel.: 9638283 69; Fax: 9638283 70. Correo-e: Roman.Ferrer@uv.es
} 
el riesgo de que los resultados y/o el valor de mercado de una entidad se vean afectados de manera adversa por los movimientos de los tipos de interés.

En la actualidad es generalmente aceptado que el riesgo de interés puede proceder de diversas fuentes. El riesgo de repreciación representa la modalidad de riesgo de interés más conocida y surge por diferencias en la sensibilidad de los activos y pasivos bancarios ante movimientos de los tipos de interés, motivadas por desequilibrios en el plazo hasta el vencimiento o los períodos de repreciación. Según esto, ante un cambio de tipos el período de repreciación medio del activo no va a coincidir con el del pasivo, de tal forma que los flujos futuros de cobros y pagos por intereses no van a variar en la misma magnitud, con la consiguiente repercusión sobre los resultados y el valor de las entidades.

Otras manifestaciones del riesgo de interés son: el riesgo de curva de rendimiento, referido a la posibilidad de que cambios no anticipados en la pendiente y en la forma de la curva de rendimientos tengan efectos perjudiciales sobre el valor y los resultados de las entidades; el riesgo de base, derivado de una correlación imperfecta entre los tipos base o de referencia de operaciones activas y pasivas con similares períodos de repreciación; y el riesgo de opcionalidad, con origen en las opciones incorporadas en numerosas operaciones activas, pasivas y fuera de balance que pueden llevar a vencimientos reales de las operaciones sustancialmente diferentes de los fijados contractualmente ${ }^{(1)}$. Por todo ello, la industria bancaria es típicamente catalogada como uno de los sectores con mayor exposición al riesgo de interés.

Además, el riesgo de interés en el ámbito del sector bancario ha cobrado una significación especial en los últimos años debido al sustancial incremento de la variabilidad de los tipos de interés y al hecho de que las fluctuaciones de los tipos de interés afectan directamente a los flujos de costes y de ingresos de las entidades bancarias. Esto ha provocado la creciente preocupación de las autoridades supervisoras por el cumplimiento de los requerimientos mínimos de capital necesarios para cubrir los riesgos bancarios, entre ellos el riesgo de interés (Acuerdos de Capital de Basilea I y II).

En este contexto, se ha desarrollado un amplio cuerpo de literatura dedicado al estudio de la exposición de las entidades financieras al riesgo de interés basado en la cuantificación de dicha exposición mediante la estimación de la sensibilidad del rendimiento de las acciones bancarias ante los movimientos de los tipos de interés.

El conocimiento del efecto de las variaciones de los tipos de interés sobre las acciones bancarias constituye una información extremadamente relevante para una adecuada gestión del riesgo de interés. De hecho, la toma de decisiones de los diferentes agentes afectados por la exposición al riesgo de interés, tales como gestores de las propias entidades financieras, inversores, autoridades supervisoras e incluso académicos, puede verse ampliamente condicionada por esta información.

(1) Ejemplos de productos con opciones incorporadas a favor de los clientes bancarios incluyen préstamos y depósitos bancarios con posibilidad de cancelación anticipada. 
Este trabajo se plantea como objetivo primordial examinar la incidencia del riesgo de interés sobre el comportamiento bursátil del sector bancario español. Para ello, se aplica metodología de tipo GARCH (heterocedasticidad condicional autorregresiva generalizada) sobre diferentes carteras de acciones bancarias construidas según el tamaño de las entidades. Su principal aportación consiste en analizar por primera vez en el caso español de manera conjunta el impacto de los cambios de los tipos de interés y de la volatilidad de tipos sobre la distribución de los rendimientos de las acciones bancarias. Específicamente, la literatura sobre riesgo de interés del mercado bursátil español [véase, por ejemplo, los trabajos de Ferrer et al. (1999), Ferrer et al. (2008) y Jareño (2006 y 2008)] ha quedado circunscrita al enfoque tradicional, limitándose al estudio del efecto de las variaciones de los tipos de interés sobre el rendimiento de las acciones, mientras que la repercusión de la volatilidad de los tipos sobre los rendimientos bursátiles ha sido ignorada.

La investigación empírica concerniente a la repercusión del riesgo de interés sobre la industria bancaria se ha centrado mayoritariamente en el mercado estadounidense. Sin embargo, la extensión de este análisis al caso español resulta especialmente interesante considerando que nuestro sistema financiero, a diferencia del de los países anglosajones, se ha caracterizado por un mayor peso relativo de los bancos respecto a los mercados de capitales en la financiación de empresas y particulares. Además, las entidades españolas responden al modelo de banca universal típico de la Europa continental, ofreciendo una amplia gama de productos y servicios financieros frente a la banca especializada predominante en el caso estadounidense. Estas diferencias confieren relevancia al estudio de la exposición al riesgo de interés del sector bancario español para comprobar si los resultados obtenidos en Estados Unidos pueden ser extrapolados a mercados con distintas características.

Asimismo, el presente trabajo también puede contribuir a esclarecer si el tamaño institucional representa un determinante significativo del grado de exposición al riesgo de interés de las entidades de crédito. En este sentido, varios motivos han sido esgrimidos para justificar las diferencias en términos de riesgo de interés existentes entre bancos de distinto tamaño.

Por un lado, los bancos más grandes pueden tener un cierto incentivo a asumir mayores niveles de riesgo debido a economías de escala en sus actividades de gestión de riesgos, a sus mayores posibilidades de diversificación de productos y clientes, a su mayor capacidad para atraer y retener personal cualificado y a su más fácil acceso a los mercados financieros globales ${ }^{(2)}$. Por otro, es también posible que los bancos de mayor dimensión muestren una actitud más agresiva hacia el riesgo a causa de un problema de riesgo moral derivado de su estatus «demasiado grande para fracasar», en virtud del cual las entidades incurren en excesivos riesgos al confiar plenamente en el respaldo del banco central en caso de graves dificultades. En contraposición, los bancos de menor tamaño presentan un comportamiento más averso al riesgo debido a su acceso más limitado a los mercados e innovaciones financieras y, en consecuencia, se centran más en la actividad bancaria tradicional y menos en la especulación.

(2) En relación con este aspecto, puede verse Amor et al. (2008). 
La evidencia empírica obtenida en este trabajo ofrece varios resultados interesantes. En primer lugar, confirma la relevancia del riesgo de interés en el ámbito del sector bancario español, poniendo de relieve que tanto los cambios de los tipos de interés como su volatilidad tienen un impacto negativo significativo sobre la distribución de rendimientos de las acciones bancarias. En segundo lugar, los tipos de interés a largo plazo se revelan como los que ejercen una influencia más significativa. En tercer y último lugar, parece existir una relación positiva entre el tamaño bancario y el grado de exposición al riesgo de interés.

El resto del trabajo queda estructurado del siguiente modo. En la Sección 2 se lleva a cabo una breve revisión de la literatura sobre riesgo de interés en el sector bancario. La Sección 3 describe la metodología aplicada, mientras que la Sección 4 presenta los datos empleados. La Sección 5 contiene los resultados obtenidos en el análisis empírico. Por último, la Sección 6 recoge las conclusiones más relevantes.

\section{REVISIÓN DE LA LITERATURA}

La incidencia del riesgo de interés sobre el rendimiento de las acciones de las entidades financieras ha sido objeto de estudio por parte de una importante corriente de literatura desarrollada a lo largo de las tres últimas décadas y centrada mayoritariamente en el caso estadounidense. El grueso de la investigación empírica ha sido efectuada en el ámbito del modelo de regresión lineal de dos índices introducido por Stone (1974) [al respecto pueden verse, entre otros, los trabajos de Flannery y James (1984), Sweeney y Warga (1986), Madura y Zarruk (1995) y Faff y Howard (1999)]. Esta formulación representa una versión ampliada del clásico modelo de mercado de Sharpe y Lintner, caracterizada por la inclusión de un factor representativo de los cambios de los tipos de interés como variable explicativa adicional junto al rendimiento de la cartera de mercado en un intento de describir más correctamente el proceso generador de rendimientos de las acciones bancarias.

Dentro de este marco, si bien es cierto que se puede apreciar una considerable variedad de metodologías aplicadas, períodos muestrales seleccionados, frecuencias de datos utilizadas, especificaciones del modelo planteadas y variables de tipo de interés empleadas, también lo es que existe un amplio consenso en torno a algunas cuestiones. En primer lugar, la gran mayoría de los trabajos documentan una relación negativa significativa entre los movimientos de los tipos de interés y el rendimiento de las acciones bancarias [véanse, por ejemplo, Flannery y James (1984), Kwan (1991) y Elyasiani y Mansur (1998)]. La explicación más frecuente apunta al habitual desequilibrio de vencimientos y duraciones entre activos y pasivos bancarios, fruto de la transformación de plazos típicamente desarrollada por las entidades financieras. A este respecto, la mayor sensibilidad del activo con respecto al pasivo frente a las variaciones de los tipos de interés debido a su mayor plazo medio hasta vencimiento provoca que en caso de subidas de tipos las entidades experimenten una reducción de su margen financiero, ya que las operaciones de pasivo tenderán a repreciarse antes que las de activo, con el subsiguiente efecto negativo sobre el valor de las entidades, mientras que en caso de bajadas de tipos se producirá el efecto contrario. En 
segundo lugar, por lo general se observa una mayor sensibilidad de las acciones bancarias ante los cambios de los tipos de interés a largo plazo que ante las variaciones de los tipos a corto. En tercer lugar, la exposición de las entidades financieras al riesgo de interés ha ido disminuyendo con el paso del tiempo, previsiblemente a causa de la mayor disponibilidad de instrumentos derivados susceptibles de ser utilizados con fines de cobertura y al desarrollo de mejores sistemas de medición y gestión del riesgo de interés.

Los trabajos iniciales sobre el riesgo de interés de las entidades bancarias se basaron en el empleo de técnicas de regresión convencionales bajo las tradicionales hipótesis de linealidad, independencia y varianza condicional constante de los rendimientos de las acciones [véanse, por ejemplo, Lynge y Zumwalt (1980), Flannery y James (1984) y Sweeney y Warga (1986)].

Con posterioridad, diversos estudios [véanse, entre otros, Yourougou (1990), Akella y Chen (1990) y Kwan (1991)] presentaron evidencia contraria a la hipótesis de varianza condicional constante, mostrando que la sensibilidad del rendimiento de las acciones bancarias ante los cambios de los tipos de interés varía de forma sustancial en el tiempo en función de las condiciones económicas, siendo especialmente acusada durante períodos de elevada variabilidad de tipos. De hecho, la no consideración del carácter variable en el tiempo del riesgo de interés puede desembocar en la obtención de estimadores sesgados e ineficientes.

En un intento de capturar explícitamente la naturaleza cambiante en el tiempo de la sensibilidad de las acciones del sector bancario ante los cambios de los tipos de interés, ha aparecido un cuerpo de trabajo más reciente basado en el empleo de metodología de tipo GARCH [véanse, por ejemplo, Song (1994), Elyasiani y Mansur (1998 y 2004), Tai (2000), Faff et al. (2005), o Joseph y Vezos (2006)]. Esta corriente asume la hipótesis de varianza condicional de los rendimientos variable en el tiempo. Además, a diferencia de la literatura previa, no se limita al estudio del efecto de las variaciones de los tipos de interés sino que también pone un especial énfasis en el impacto de la volatilidad de tipos sobre la distribución de rendimientos de las acciones bancarias.

En concreto, Song (1994) es el trabajo pionero en la aplicación de la metodología ARCH (heterocedasticidad condicional autorregresiva) en el sector bancario, demostrando la idoneidad de este enfoque. Posteriormente, Elyasiani y Mansur (1998) y Ryan y Worthington (2004) emplean modelos GARCH-M (GARCH en media) de tipo univariante para examinar tanto el efecto de las variaciones como de la volatilidad de los tipos de interés sobre la distribución de rendimientos de diversas carteras bancarias en los mercados estadounidense y australiano, respectivamente. Siguiendo un enfoque parecido, Joseph y Vezos (2006) utilizan un modelo EGARCH (GARCH exponencial) univariante en el mercado estadounidense. Por su parte, Tai (2000) y Faff et al. (2005) proponen modelos GARCH-M multivariantes para examinar el impacto dual de los cambios de los tipos de interés y de la volatilidad de tipos sobre los rendimientos de carteras bancarias en los mercados estadounidense y australiano, respectivamente. De forma similar, Elyasiani y Mansur (2004) aplican un modelo GARCH multivariante en el sector bancario estadounidense. El Cuadro 1 contiene un resumen de los principales objetivos y resultados de estos trabajos. 


\begin{tabular}{|c|c|c|c|c|c|c|c|}
\hline \multirow{6}{*}{ 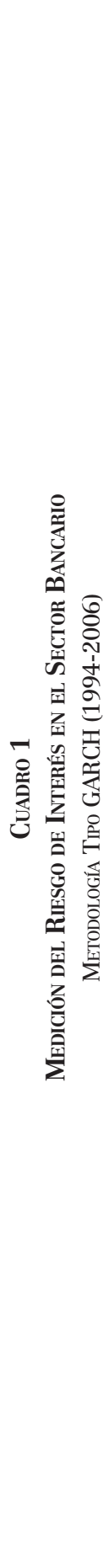 } & 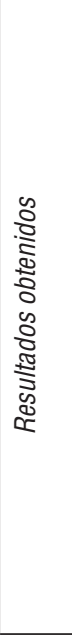 & 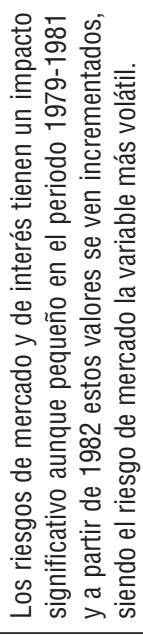 & 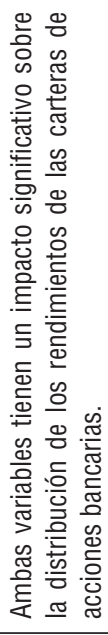 & 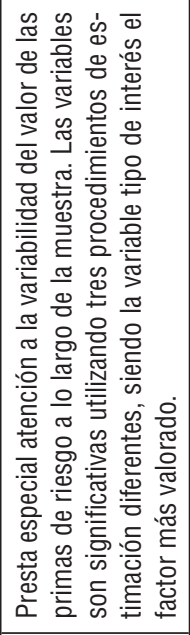 & 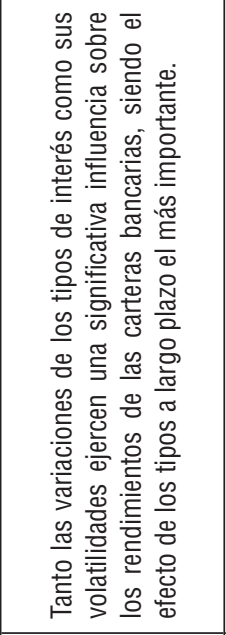 & 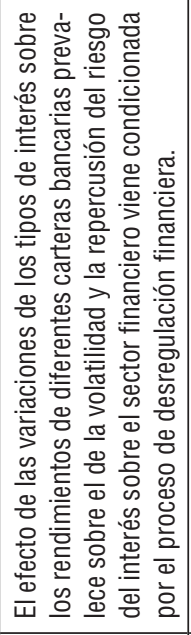 & 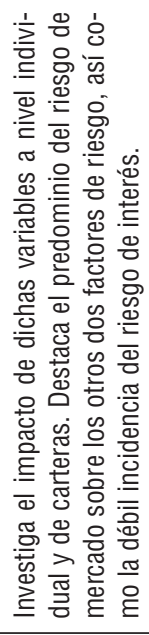 \\
\hline & 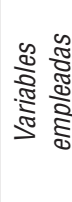 & 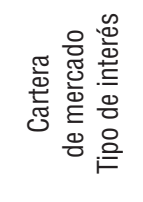 & 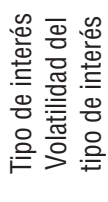 & 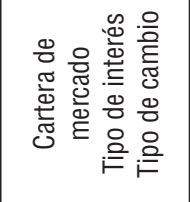 & 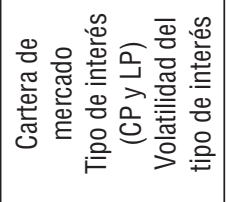 & 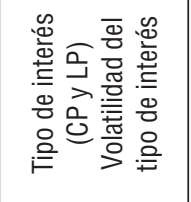 & 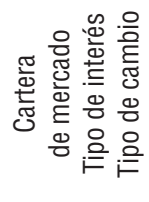 \\
\hline & 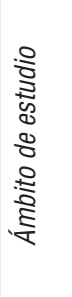 & 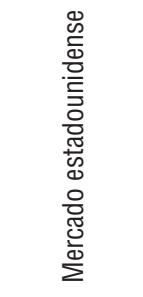 & 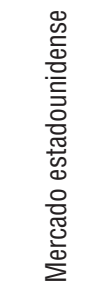 & 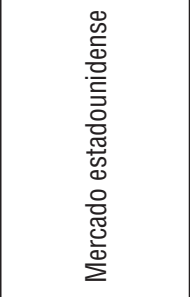 & 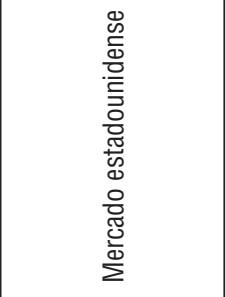 & 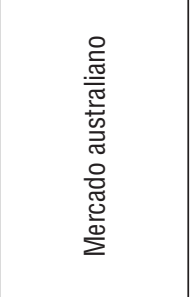 & 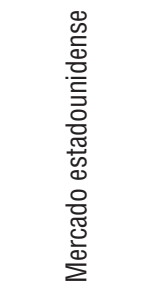 \\
\hline & 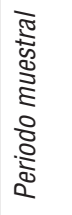 & $\begin{array}{l}\hat{o} \\
\frac{0}{1} \\
\frac{1}{1} \\
\text { o } \\
\frac{1}{1}\end{array}$ & $\begin{array}{l}\text { శ్ } \\
\frac{\sigma}{1} \\
\text { 함 }\end{array}$ & 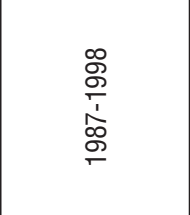 & 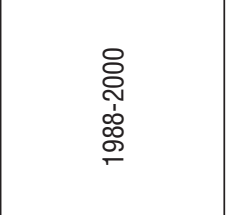 & 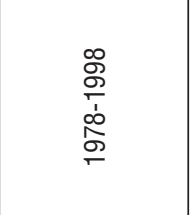 & $\begin{array}{l}\text { ᄋे } \\
\text { ते } \\
\text { ᄋे } \\
\text { ᄋ }\end{array}$ \\
\hline & $\begin{array}{l}\frac{\pi}{5} \\
\frac{1}{2} \\
\frac{5}{2} \\
\frac{5}{2}\end{array}$ & 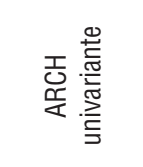 & 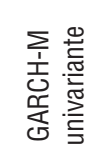 & 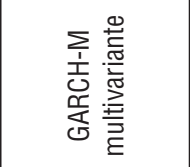 & 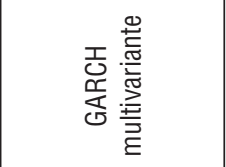 & 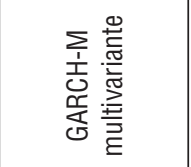 & 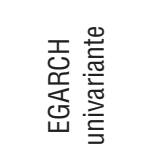 \\
\hline & $\frac{\mathscr{2}}{\pi}$ & 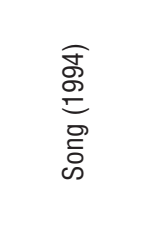 & 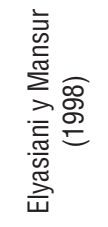 & 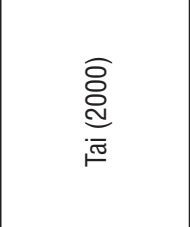 & 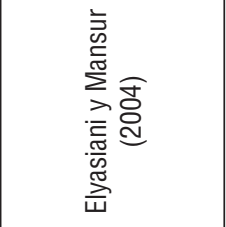 & 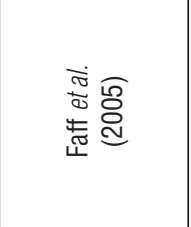 & 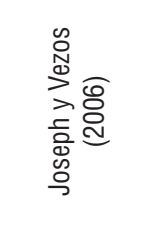 \\
\hline
\end{tabular}


En lo que concerniente al caso español, la evidencia disponible relativa al riesgo de interés del sector financiero es considerablemente inferior al caso estadounidense, si bien se pueden destacar algunos trabajos interesantes. Al respecto, con el propósito de determinar la relevancia del riesgo de interés en el comportamiento de los distintos sectores bursátiles Ferrer et al. (1999) utilizan un modelo unifactorial cuya única variable explicativa recoge el impacto de los cambios de tipos sobre los rendimientos bursátiles sectoriales. Por su parte, Ferrer et al. (2008) examinan la exposición al riesgo de interés de las empresas españolas, también a nivel sectorial, en el ámbito del modelo bifactorial de Stone centrándose exclusivamente en el efecto de los cambios de los tipos de interés y empleando técnicas de regresión móvil. En ambos estudios se constata que el sector bancario constituye, junto con los sectores de construcción y eléctrico, uno de los más afectados por los movimientos de los tipos de interés.

Desde una perspectiva distinta, Jareño (2006 y 2008) propone una extensión del clásico modelo de Stone (1974) y un enfoque híbrido entre el modelo de Stone y el modelo de tres factores de Fama y French (1993), respectivamente, para analizar por separado el efecto de los cambios de los tipos de interés reales y de la tasa de inflación esperada sobre el mercado de acciones a nivel sectorial. En ambos trabajos se pone de manifiesto que el sector bancario español presenta una significativa sensibilidad negativa ante las variaciones de los tipos de interés reales, mientras que la inflación esperada no tiene un impacto significativo.

\section{METODOLOGÍA}

La formulación tradicionalmente empleada para medir la sensibilidad de las acciones bancarias ante los movimientos de los tipos de interés es el modelo de dos índices introducido por Stone (1974), cuya expresión analítica es la siguiente:

$$
R_{i t}=\alpha_{i}+\lambda_{i} R_{m t}+\theta_{i} \Delta I_{t}+\varepsilon_{i t}
$$

donde $R_{i t}$ denota el rendimiento de la acción de la entidad bancaria $i$ en el periodo $t$, el $R_{m t}$ el rendimiento de la cartera de mercado en el periodo $t, \lambda_{i}$ la sensibilidad del rendimiento de la acción $i$ ante los movimientos generales del mercado, $\Delta I_{t}$ los cambios del tipo de interés en el periodo $t, \theta_{i}$ la sensibilidad del rendimiento de la acción $i$ ante las variaciones de los tipos de interés independientemente del efecto que estas variaciones tienen sobre el mercado bursátil en su conjunto y, finalmente, $\varepsilon_{i t}$ indica el término de error.

El coeficiente $\theta_{i}$ puede ser visto como una estimación de la duración de la acción bancaria $i$ y, por consiguiente, representa un indicador del grado de exposición al riesgo de interés de la entidad financiera $i$.

Dada la habitual tendencia a la agrupación de la volatilidad (volatility clustering) en la mayor parte de las series temporales financieras, el modelo contenido en (1) ha sido ampliado con el fin de capturar el carácter cambiante en el tiempo de la varianza condicional del rendimiento de las acciones bancarias. En este contexto, la metodología GARCH ha sido expresamente desarrollada para ocuparse de los potenciales problemas derivados de 
la presencia de heterocedasticidad condicional. Esta aproximación combina una especificación relativamente simple con una memoria larga del proceso de volatilidad y dentro de la misma se encuadran los modelos ARCH introducidos por Engle (1982), así como los procesos GARCH propuestos por Bollerslev (1986) y las extensiones ARCH-M y GARCH-M de Engle et al. (1987).

Específicamente, la rama de investigación sobre riesgo de interés en el sector bancario con mayor protagonismo en la literatura reciente es la basada en la aplicación de los modelos GARCH-M [véanse a este respecto, por ejemplo, Elyasiani y Mansur (1998), Ryan y Worthington (2004), Tai (2000) o Faff et al. (2005)]. El rasgo distintivo de estos procesos es su capacidad para capturar la dinámica variable en el tiempo de la prima de riesgo, así como la posible relación existente entre riesgo y rendimiento esperado.

El modelo aquí propuesto es una versión extendida de un modelo GARCH(1,1)-M univariante y viene descrito por el siguiente sistema de ecuaciones:

$$
\begin{gathered}
R_{i t}=\omega_{i}+\lambda_{i} R_{m t}+\theta_{i} \Delta I_{t}+\gamma_{i} \log h_{i t}+\varepsilon_{i t} \\
h_{i t}=\alpha_{0}+\alpha_{1} \varepsilon_{i t-1}^{2}+B h_{i t-1}+\delta_{i} V C I_{t-1} \\
\varepsilon_{i t} \mid \Omega_{t-1} \sim N\left(0, h_{i t}\right)
\end{gathered}
$$

donde $h_{i t}$ refleja la varianza condicional del rendimiento de la acción $i$ en el período $t, V C I_{t-1}$ es la volatilidad del tipo de interés en el período $t$ - 1 y $\varepsilon_{i t}$ es un término de error gaussiano de media cero y varianza $h_{i t}$, dependiente del conjunto de información relevante disponible en $t-1, \Omega_{t-1}$. Finalmente, $\omega_{i}, \lambda_{i}, \theta_{i}, \gamma_{i}, \alpha_{0}, \alpha_{1}, \beta, \delta_{i}$ y son los parámetros a estimar. Los mismos deben satisfacer las condiciones de estacionariedad, de tal forma que y $\alpha_{0}, \alpha_{1}, \beta \geq 0$ y $\alpha_{1}+\beta<1$.

En lo concerniente a la especificación planteada, se ha considerado más oportuno usar un modelo GARCH univariante que un enfoque multivariante. Ello obedece a que en el caso concreto del presente estudio parece claro desde un punto de vista económico que la causalidad va desde el riesgo de interés y el riesgo de mercado en dirección al rendimiento de las acciones bancarias, mientras que es ciertamente difícil justificar un efecto de retroalimentación del rendimiento de las acciones bancarias sobre el rendimiento de mercado y las fluctuaciones de los tipos de interés. Es por ello que la modelización adicional incorporada por un proceso multivariante para analizar posibles efectos de transmisión de volatilidad entre la variable dependiente y las variables independientes no parece necesaria para el estudio del riesgo de interés del sector bancario. Por consiguiente, la mayor sencillez operativa junto con el intento de evitar problemas en la estimación derivados del incremento exponencial del número de parámetros a estimar en los modelos multivariantes representan argumentos de peso a favor del empleo de una especificación GARCH univariante. Adicionalmente, la presencia de posibles efectos asimétricos en el impacto de los cambios positivos y negativos de los tipos de interés sobre las acciones bancarias ha sido examinada. Los resultados contrarios a la existencia de asimetrías significativas justifican la no utilización de modelos GARCH asimétricos.

El empleo de un proceso GARCH-M de orden $(1,1)$ viene apoyado por numerosos trabajos empíricos que muestran que los modelos GARCH $(1,1)$ capturan adecuadamente el com- 
portamiento heterocedástico condicional de gran cantidad de series temporales financieras. En este sentido, se pueden destacar, entre otros, los trabajos de Baillie y DeGennaro (1990), Ryan y Worthington (2004), Amigo y Rodríguez (2007) o García Blandón (2008).

El modelo propuesto presenta características muy similares a los empleados por Elyasiani y Mansur (1998) y Ryan y Worthington (2004). La utilización de estos modelos como referencia obedece a diversas razones. Por un lado, permiten examinar conjuntamente el impacto de los cambios de los tipos de interés y de la volatilidad de tipos sobre la distribución de rendimientos de las acciones bancarias en el marco de un proceso GARCH $(1,1)$ univariante, con las consiguientes ventajas sobre los modelos multivariantes en términos de sencillez operativa. Por otro, el uso de una especificación GARCH «en media» permite incorporar el efecto de la volatilidad bancaria como un determinante adicional del rendimiento de las acciones capturando, por tanto, el posible efecto de retroalimentación entre riesgo y rendimiento esperado consistente con modelos de valoración de activos como el CAPM o el APT.

Como se puede apreciar, la ecuación de la media condicional del modelo formulado, recogida en (2), incluye como variable explicativa adicional, junto al rendimiento de la cartera de mercado y los cambios de los tipos de interés ya utilizados por Stone, la varianza del rendimiento de la acción bancaria en cuestión. En relación a esta última, su especificación en forma logarítmica sigue el planteamiento de Engle et al. (1987), para quienes el logaritmo de la varianza es una mejor representación del riesgo que la propia varianza o la desviación típica. A su vez, el parámetro conecta explícitamente la varianza condicional con la media condicional del rendimiento del activo en cuestión y es usualmente interpretado como el coeficiente de aversión relativa al riesgo.

En lo tocante a la ecuación de la varianza condicional, recogida en (3), la estructura típica de los procesos GARCH ha sido ampliada al modelizar la varianza condicional del rendimiento de las acciones bancarias como función de la volatilidad de los tipos de interés retardada un período. Así, el parámetro $\delta_{i}$ determina la significatividad de la volatilidad de los tipos de interés.

En este contexto, si bien hasta el momento la literatura se ha centrado en el impacto de las variaciones de los tipos de interés, con un nivel de atención muy superior al otorgado a la incidencia de la volatilidad de los tipos de interés, debe tenerse en cuenta que el efecto de la volatilidad de tipos sobre la distribución de los rendimientos de las acciones bancarias también constituye un aspecto de indudable relevancia. En concreto, su estudio va a permitir alcanzar un mayor grado de comprensión del comportamiento de las entidades financieras en respuesta a las fluctuaciones de los tipos de interés.

Específicamente, tal y como señalan Elyasiani y Mansur (1998) la incorporación del impacto de la volatilidad de tipos en el modelo resulta importante desde varias perspectivas. Por un lado, desde un punto de vista macroeconómico la volatilidad de los tipos de interés contiene información esencial sobre el nivel de volatilidad global en los mercados financieros, ya que esta variable refleja la incertidumbre existente sobre la política monetaria y la efectividad de la autoridad supervisora en la consecución de sus objetivos. Por otro, la volatilidad de tipos también influye sobre la volatilidad del rendimiento de las acciones 
bancarias a nivel microeconómico. A este respecto, Deshmukh et al. (1983) demostraron que, ante un incremento de la incertidumbre relativa a los tipos de interés, las entidades bancarias intentan protegerse reduciendo su nivel de exposición a dicho riesgo, lo que acaba traduciéndose en una volatilidad más baja del rendimiento de sus acciones. Este efecto se ve reforzado por un posible problema de agencia con los gestores bancarios, quienes, preocupados por mantener su puesto de trabajo, tienen un claro incentivo a reducir los riesgos financieros para contrarrestar el aumento de la incertidumbre sobre los tipos de interés.

Junto a todo lo anterior, existen varios aspectos relevantes concernientes a la estimación del modelo que merecen ser comentados. Una primera cuestión atañe a la posible colinealidad entre las series de variaciones de los tipos de interés y rendimiento de la cartera de mercado, derivada de una fuerte correlación entre ambas que puede provocar serios problemas en la estimación. Dada la significativa correlación negativa típicamente observada en el caso español entre ambas variables, se ha considerado conveniente eliminar dicha correlación mediante la aplicación de un procedimiento de ortogonalización. Dado que el objetivo central del trabajo es el análisis del efecto del riesgo de interés sobre las entidades financieras, se ha optado por ortogonalizar el rendimiento del mercado siguiendo un esquema idéntico al empleado por Lynge y Zumwalt (1980), Flannery y James (1984) y Czaja et al. (2006).

Un segundo aspecto a destacar concierne a la elección de la variable de tipo de interés concreta a emplear en el análisis empírico. Al respecto, los tipos de interés a largo plazo, y en particular el tipo a diez años, constituyen la variable más frecuentemente usada en la literatura $^{(3)}$. Ello se debe a que los tipos a largo plazo son los que incorporan en mayor medida las expectativas de los agentes sobre el futuro y los que determinan en la práctica el coste de la financiación, convirtiéndose, por tanto, en los que ejercen una mayor influencia sobre las decisiones de inversión empresarial y la evolución de la actividad económica. No obstante, y con el objeto de dotar de una mayor robustez a los resultados, los tipos de interés a corto plazo y el margen o spread entre los tipos a largo y a corto plazo han sido también empleados. En relación a los tipos de interés a corto plazo, se ha escogido un tipo del mercado interbancario dado que durante los últimos años este mercado se ha convertido en una referencia esencial para las entidades financieras españolas debido tanto al espectacular incremento de las operaciones de activo y pasivo referenciadas a tipos interbancarios como a que dicho mercado ha sido utilizado con profusión por las entidades para financiar sus operaciones de activo, principalmente del segmento hipotecario, en el marco del boom de la vivienda en España. A su vez, el margen entre tipos refleja la pendiente de la curva de tipos, pudiendo actuar como un indicador de la evolución del ciclo económico.

Una última cuestión hace referencia a la estimación de la volatilidad de los tipos de interés. Tal y como señalan Poon y Granger (2003), una gran cantidad de trabajos han usado modelos de la familia GARCH para estimar la volatilidad de los tipos de interés, obteniendo generalmente evidencia de persistencia en la volatilidad. En este contexto, siguiendo a El-

(3) Como ejemplos de utilización de los tipos a diez años pueden verse, entre otros, los trabajos de Sweeney y Warga (1986), Elyasiani y Mansur (1998), Tai (2000), Ryan y Worthington (2004) o Faff et al. (2005). 
yasiani y Mansur (1998) y Ryan y Worthington (2004) la volatilidad de cada tipo de interés ha sido estimada por separado a través de la varianza condicional de dicho tipo generada con un proceso GARCH y después incorporada a la ecuación de la varianza condicional.

\section{DATOS EMPLEADOS}

El período de análisis se extiende desde enero de 1993 hasta diciembre de 2005, abarcando un intervalo temporal en el que los tipos de interés han variado sustancialmente dentro de una marcada tendencia general a la baja. La base de datos utilizada está compuesta por series históricas de frecuencia mensual de precios bursátiles y de tipos de interés.

El empleo de datos mensuales permite trabajar de una forma más manejable con períodos muestrales de considerable amplitud, tendiendo a reflejar adecuadamente los movimientos a largo plazo en la volatilidad. Además, los datos de frecuencia más alta (por ejemplo diaria) contienen una mayor cantidad de ruido atribuible a factores de tipo idiosincrásico, lo que reduce la eficiencia de las estimaciones y dificulta la obtención de una relación significativa entre riesgo de interés y rendimientos bancarios. De hecho, numerosos trabajos en este contexto como los de Song (1994), Elyasiani y Mansur (1998), Faff y Howard (1999), y Faff et al. (2005), han usado también datos mensuales.

En relación a los datos bursátiles, se han utilizado las series de precios de cierre mensuales, ajustados por ampliaciones de capital, splits y dividendos, de las acciones de todos los bancos negociados en el Sistema de Interconexión Bursátil Español (SIBE) durante el período muestral (23 entidades en total). El rendimiento mensual de cada acción bancaria ha sido calculado como la variación relativa de su valor de cierre a final de mes entre dos observaciones consecutivas. Estos datos incluyen el conjunto de bancos negociados en el SIBE durante, al menos, un período anual y no sólo los cotizados durante todo el período de estudio, apareciendo así bancos que han sido excluidos del mercado por varias razones (compra, absorción, etc.) y otros que han sido creados (fusión) a lo largo del período muestral. Este procedimiento de selección muestral permite utilizar todos los datos disponibles en cada período anual, minimizando el posible sesgo de supervivencia y maximizando el tamaño de la muestra con objeto de mejorar la eficiencia de la estimación. El Cuadro 2 contiene el listado de bancos considerados, mostrando para cada uno su ticker bursátil junto con el volumen total de activos medio durante el período de estudio y el número de observaciones disponible.

A su vez, la cartera de mercado utilizada constituye una versión modificada del Índice General de la Bolsa de Madrid (IGBM). Específicamente, se ha empleado un índice de mercado alternativo del que no forman parte los bancos con la finalidad de obtener una serie de rendimientos de la cartera de mercado lo más exógena posible a los rendimientos de las carteras bancarias, dado el gran peso del sector bancario dentro del IGBM ${ }^{(4)}$. Todos los datos bursátiles necesarios para confeccionar el índice de mercado alternativo proceden de Sociedad de Bolsas, S.A.

(4) A pesar de la no consideración de los bancos en el índice de mercado modificado, la correlación de este índice con respecto al IGBM original durante el periodo de estudio considerado es 0,95. 


\section{Cuadro 2}

LISTADO DE BANCOS Y COMPOSICIÓN DE LAS CARTERAS

\begin{tabular}{|c|c|c|c|c|c|c|c|}
\hline CARTERAS & TICKER & $\begin{array}{l}\text { VolUMEN ACTIVO } \\
\text { (MILES E) }\end{array}$ & OBS. & & TICKER & $\begin{array}{l}\text { VolUMEN ACTIVO } \\
\text { (MILES E) }\end{array}$ & OBS. \\
\hline \multicolumn{8}{|l|}{ Cartera G } \\
\hline $\begin{array}{l}\text { Banco Santander } \\
\text { Central Hispano }\end{array}$ & $\mathrm{BSCH}$ & 369.124 .995 & 81 & $\begin{array}{l}\text { Banco Bilbao } \\
\text { Vizcaya }\end{array}$ & BBV & 100.026 .979 & 85 \\
\hline $\begin{array}{l}\text { Banco Bilbao Vizcaya } \\
\text { Argentaria }\end{array}$ & BBVA & 297.433 .664 & 71 & Argentaria & ARG & 69.998 .972 & 80 \\
\hline Banco Santander & SAN & 113.404 .303 & 75 & $\begin{array}{l}\text { Banco Central } \\
\text { Hispano }\end{array}$ & $\mathrm{BCH}$ & 68.793 .146 & 75 \\
\hline \multicolumn{8}{|l|}{ Cartera M } \\
\hline Banesto & BTO & 42.332 .585 & 156 & Bankinter & BKT & 15.656 .910 & 156 \\
\hline Banco Exterior & EXT & 32.130 .967 & 51 & Banco Pastor & PAS & 8.789 .945 & 156 \\
\hline $\begin{array}{l}\text { Banco Popular } \\
\text { Español }\end{array}$ & POP & 29.548 .620 & 156 & Banco Atlántico & ATL & 7.591 .378 & 138 \\
\hline Banco Sabadell & $\mathrm{SAB}$ & 26.686 .670 & 56 & & & & \\
\hline \multicolumn{8}{|l|}{ Cartera $P$} \\
\hline Banco Zaragozano & ZRG & 4.597 .099 & 130 & Banco Galicia & GAL & 1.726 .563 & 156 \\
\hline Banco Valencia & BVA & 4.213 .420 & 156 & $\begin{array}{l}\text { Banco de } \\
\text { Vasconia }\end{array}$ & VAS & 1.330 .458 & 156 \\
\hline Banco Guipuzcoano & GUI & 4.082 .463 & 156 & $\begin{array}{l}\text { Banco de } \\
\text { Vitoria }\end{array}$ & VIT & 875.974 & 62 \\
\hline Banco Andalucía & AND & 3.521 .838 & 156 & $\begin{array}{l}\text { Banco Crédito } \\
\text { Balear }\end{array}$ & CBL & 854.972 & 156 \\
\hline Banco Herrero & HRR & 2.624 .824 & 95 & Banco Alicante & ALI & 835.576 & 64 \\
\hline Banco de Castilla & CAS & 2.151 .742 & 156 & Banco Simeón & SIM & 686.451 & 67 \\
\hline
\end{tabular}

Este cuadro muestra la lista de bancos considerados y su distribución en carteras grande $(G)$, mediana $(M)$ y pequeña $(P)$ según un criterio de tamaño (volumen total de activos).

Por otro lado, el tanto interno de rendimiento medio mensual de las obligaciones del Estado a diez años negociadas en el mercado secundario de deuda pública anotada y la media mensual del tipo de interés a tres meses del mercado interbancario han sido tomados como variables representativas de los tipos a largo y a corto plazo, respectivamente. Por su parte, el spread de tipos ha sido calculado como la diferencia entre los tipos de interés a diez años y a tres meses. Estos datos han sido obtenidos de las series históricas de mercados financieros publicadas por el Banco de España.

Como se ha comentado antes, la volatilidad de los tipos de interés ha sido medida a través de su varianza condicional modelizada mediante un proceso de tipo GARCH. En particular, tras convertir cada una de las variables de tipos de interés en una serie estacionaria e incorporar en la ecuación de la media condicional el número de términos autorregresivos necesario para garantizar la ausencia de autocorrelación, se ha comprobado que el modelo más apropiado para la ecuación de la varianza condicional de los tipos de interés es siempre un GARCH $(1,1)$. El Gráfico 1 muestra la evolución temporal de las distintas series de tipos de interés en niveles. 


\section{GráFICo 1}

EvOLUCIÓN DE LOS DIFERENTES TIPOS DE INTERÉS

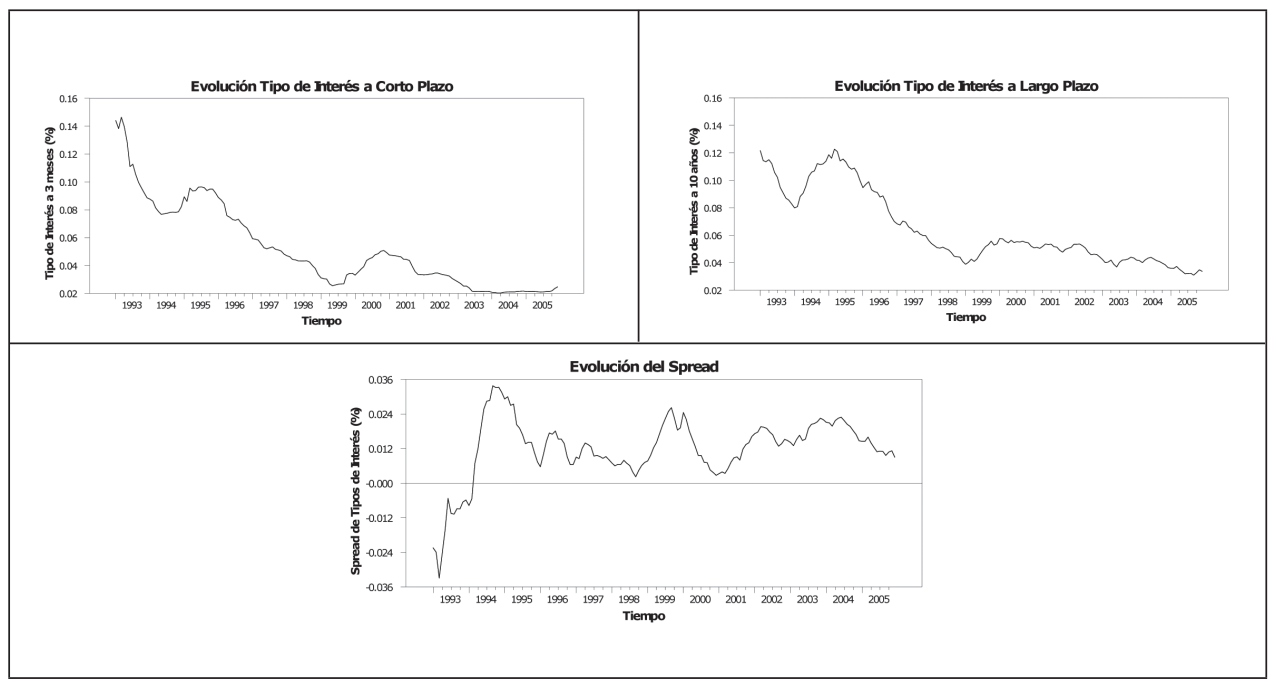

En sintonía con la práctica habitual en la literatura y a fin de comprobar si existe algún tipo de relación sistemática entre el tamaño de las entidades financieras y su grado de exposición al riesgo de interés, el estudio se ha llevado a cabo a nivel de carteras de acciones bancarias construidas según criterios de tamaño en lugar de trabajar con títulos individuales. De esta forma, los bancos de la muestra han sido clasificados en tres carteras en función de su volumen total de activos medio. Así, se ha creado una cartera de bancos grandes (Cartera $\mathrm{G}$ en lo sucesivo), una cartera de bancos medianos (Cartera M) y una cartera de bancos pequeños (Cartera P), cuya composición, recogida en el Cuadro 2, se ha mantenido inalterada durante el periodo de estudio. En concreto, la Cartera G está compuesta por aquellas entidades con un volumen total de activos superior a 50.000 millones de euros, propiciando la inclusión en la misma de los dos grandes conglomerados bancarios españoles (Grupos Banco Santander y BBVA). A su vez, la cartera M está formada por aquellas entidades con un volumen de activos comprendido entre 5.000 y 50.000 millones de euros. Un total de siete entidades, representativas de la banca mediana española (Banesto, Banco Popular, Banco Sabadell, Bankinter, etc.) integran esta categoría. Por último, la Cartera $\mathrm{P}$, constituida por los bancos con activos totales por debajo de 5.000 millones de euros, se nutre de los doce restantes bancos de menor dimensión.

La serie de rendimientos mensuales de cada cartera bancaria ha sido obtenida como la media aritmética ponderada de los rendimientos mensuales de las acciones integrantes de la cartera en cuestión. Como factor de ponderación se ha empleado el porcentaje que representa la capitalización bursátil de cada banco respecto de la capitalización bursátil total de la cartera al final de cada periodo. El Gráfico 2 muestra la evolución temporal de los rendimientos mensuales de las Carteras G, M y P en comparación con el rendimiento mensual de la cartera de mercado. 


\section{GráFICO 2}

Rendimientos mensuales de las carteras G, M y P vs Rendimiento de la cartera de mercado

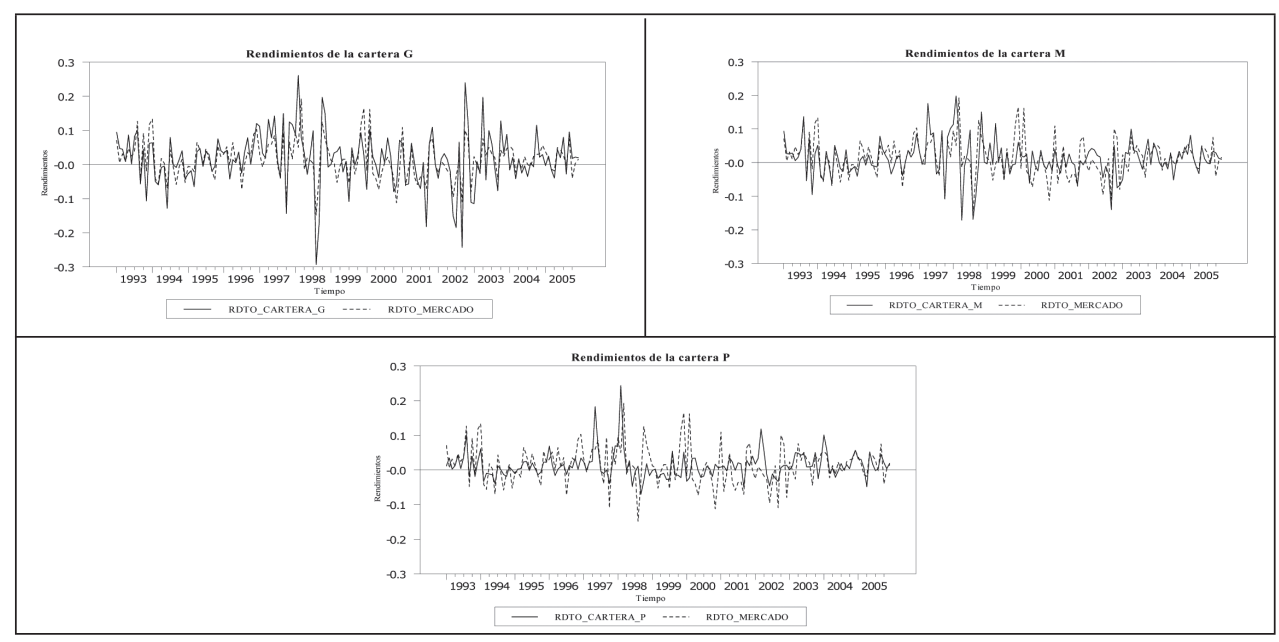

La formación de carteras proporciona una manera eficiente de resumir una gran cantidad de información sobre el comportamiento de las acciones, con la ventaja añadida de suavizar el ruido presente en los datos, debido principalmente a shocks transitorios en las compañías individuales. En contraposición, el empleo de carteras puede enmascarar las diferencias existentes entre empresas individuales. No obstante, para el tipo de análisis aquí efectuado las ventajas asociadas a la construcción de carteras parecen superar el inconveniente señalado y buena prueba de ello es la proliferación de trabajos basados en el empleo de carteras bancarias [véase, por ejemplo, Song (1994), Elyasiani y Mansur (1998 y 2004), Faff et al. (2005) o Joseph y Vezos (2006)] ${ }^{(5)}$.

\section{RESULTADOS EMPÍRICOS}

\subsection{Propiedades de los datos}

De forma preliminar, con el propósito de comprobar la idoneidad de la metodología GARCH en el presente estudio se ha examinado las propiedades de normalidad, ruido blanco, asimetría y curtosis de las series de rendimientos de las carteras de acciones bancarias. Los resultados obtenidos aparecen en la Tabla 1.

El aspecto más destacable es la presencia de asimetría y curtosis significativas en los rendimientos de las carteras bancarias, sugiriendo que estas series no siguen una distribución normal, evidencia reforzada por el claro rechazo de la hipótesis de normalidad con el test de Jarque-Bera. Además, los estadísticos del contraste de Ljung-Box sobre las series de rendimientos al cuadrado son estadísticamente significativos para las tres carteras banca-

(5) No obstante, se ha estimado también un modelo GARCH para cada una de las acciones bancarias, comprobándose que los resultados son análogos a los obtenidos con carteras. Esta evidencia no ha sido incluida por motivos de espacio, pero se encuentra disponible para cualquier lector interesado. 
rias, si bien en menor grado para la Cartera P. Este resultado de dependencia no lineal es típicamente asociado a la presencia de heterocedasticidad condicional. Así pues, la evidencia obtenida sugiere que el enfoque GARCH puede ser un marco apropiado para analizar el riesgo de interés de las acciones bancarias.

TABLA 1

PrinCIPALES ESTAdísticos DESCRIPTIVOS DE LAS SERIES DE RENDIMIENTOS DE LAS CARTERAS BANCARIAS

\begin{tabular}{|c|c|c|c|}
\hline & \multicolumn{3}{|c|}{ Carteras Bancarias Ponderadas } \\
\hline & Cartera G & Cartera M & Cartera $P$ \\
\hline Observaciones & 156 & 156 & 156 \\
\hline Media & 0,0165 & 0,0116 & 0,0130 \\
\hline Varianza muestral & 0,0064 & 0,0029 & 0,0014 \\
\hline Mínimo & $-0,2926$ & $-0,1711$ & $-0,0701$ \\
\hline Máximo & 0,2611 & 0,1982 & 0,2435 \\
\hline Asimetría & $-0,4465^{\star *}$ & $-0,0028$ & $2,2059^{\star * *}$ \\
\hline Curtosis & $5,1566 * * *$ & $5,3475^{\star * *}$ & $13,4299 * * *$ \\
\hline$J B$ & $35,4181^{* * *}$ & $35,8218^{\star * *}$ & $833,6212^{\star * *}$ \\
\hline $\mathrm{Q}(12)$ & 9,6344 & 9,8951 & $29,2857^{\star * *}$ \\
\hline $\mathrm{Q}(24)$ & 12,5575 & 19,5186 & $35,6335^{\star}$ \\
\hline$Q(36)$ & 24,3749 & 27,1294 & $50,6139 *$ \\
\hline$Q(12)$ & $49,5900^{\star * *}$ & $95,9229^{\star * *}$ & $25,9366^{* *}$ \\
\hline$Q(24)$ & $61,6832^{* * *}$ & $109,5708^{* * *}$ & 28,3502 \\
\hline$Q(36)$ & $100,7131^{* * *}$ & $145,1550^{\star * *}$ & 32,6492 \\
\hline
\end{tabular}

$J B$ es el estadístico Jarque-Bera que contrasta la hipótesis nula de normalidad de los rendimientos de las carteras bancarias. $Q(n)$ es el estadístico Ljung-Box para $n$ retardos que contrasta la presencia de correlación serial de orden $n$. Finalmente, ${ }^{* * *},{ }^{* *} \mathrm{y}^{*}$ denotan significatividad a los niveles del $1 \%, 5 \%$ y $10 \%$, respectivamente.

Adicionalmente, a fin de determinar si las diferentes series son estacionarias, se han aplicado los contrastes de raíces unitarias Dickey-Fuller aumentado y Phillips-Perron. Los resultados indican que todas las series de rendimientos son estacionarias en niveles, al igual que el spread entre tipos de interés, mientras que las series de tipos de interés a diez años y a tres meses en niveles presentan una raíz unitaria, lo que justifica el uso de sus primeras diferencias ${ }^{(6)}$.

\subsection{INTERPRETACIÓN DE LOS RESULTADOS}

Los resultados de la estimación del modelo propuesto para cada una de las carteras de acciones bancarias utilizando las diferentes variables alternativas de tipos de interés aparecen en la Tabla 2. En este sentido, debe tenerse en cuenta que la escasa variabilidad de los rendimientos de la Cartera P, unida a los ya de por sí más débiles indicios de heterocedasticidad condicional hallados en la misma, provocan que la inclusión de la varianza condicional de los tipos de interés genere problemas en la estimación del modelo propuesto para el caso de la Cartera P. Por esta razón, se ha optado por excluir la volatilidad de los tipos de interés de la ecuación de la varianza condicional en el modelo finalmente estimado para la Cartera P.

(6) Estos resultados no se han incluido en el trabajo por cuestiones de espacio si bien pueden ser solicitados a los autores. 


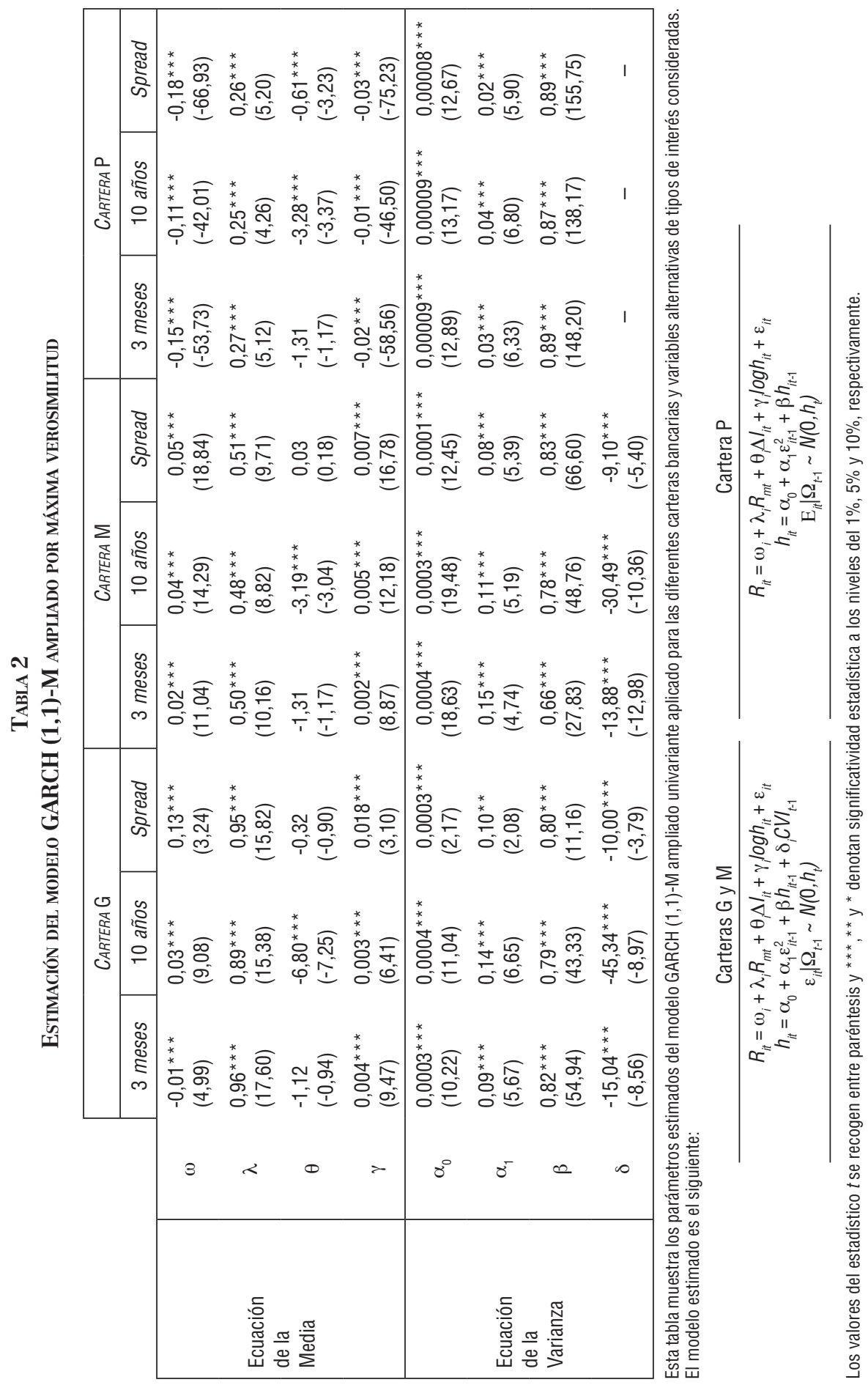


En línea con lo esperado, la estimación del modelo para las tres carteras de acciones bancarias permite constatar cómo el parámetro $\lambda$, representativo de la sensibilidad del rendimiento de la cartera bancaria en cuestión ante los movimientos generales del mercado, es positivo y significativo a un nivel del 1\% en todos los casos y con independencia de la variable de tipos de interés considerada. Dicho parámetro adopta siempre valores inferiores a la unidad y su cuantía desciende conforme disminuye el tamaño de la cartera. Así pues, este resultado indica que el riesgo de mercado o sistemático constituye un factor explicativo relevante del comportamiento de las acciones bancarias.

Asimismo, en el sector bancario español parece existir una relación directa entre el tamaño de las entidades y su nivel de riesgo de mercado. Este resultado no se ve en absoluto influenciado por el peso que puedan tener las acciones bancarias dentro del mercado bursátil en su conjunto, puesto que éstas han sido expresamente excluidas del índice alternativo de mercado utilizado. Una explicación plausible de esta vinculación positiva apunta a que los bancos más grandes tienen mayores oportunidades para diversificar sus carteras de activos, operando generalmente en un amplio rango de zonas y sectores económicos, y además participan más activamente en operaciones fuera de balance arriesgadas (por ejemplo, mediante posiciones en derivados). Por ello, su comportamiento está más correlacionado con los shocks que afectan al mercado en general. Frente a esto, los bancos de dimensión más reducida suelen presentar una menor diversificación geográfica y de productos y un comportamiento bursátil más regido por factores de tipo idiosincrásico, lo que se traduce en una menor conexión con las fluctuaciones del mercado. A su vez, el hecho de que $\lambda$ sea menor que la unidad parece sugerir que las carteras bancarias presentan, independientemente del tamaño institucional, un nivel de riesgo sistemático inferior al del mercado de acciones en su conjunto.

En relación al impacto de los cambios en el nivel de los tipos de interés, el parámetro $\theta$ es negativo prácticamente siempre, si bien sólo resulta significativo a los niveles usuales para las tres carteras al utilizar las variaciones de los tipos a diez años. Además, los movimientos de los tipos de interés a largo plazo son los que ejercen una influencia más fuerte sobre las acciones bancarias en términos de magnitud y significatividad estadística, de forma consistente con los resultados de la mayor parte de la literatura centrada en el riesgo de interés del sector bancario [véanse, por ejemplo, los trabajos de Madura y Zarruk (1995), Elyasiani y Mansur (1998 y 2004) y Bartram (2002)]. Frente a esto, las variaciones de los tipos de interés a corto plazo y el spread de tipos no tienen un efecto significativo sobre el rendimiento de las tres carteras bancarias, exceptuando el spread en el caso de la Cartera P.

Asimismo, también parece existir una relación directa entre el grado de sensibilidad ante los cambios de los tipos de interés y el tamaño de las entidades bancarias. Una posible explicación puede ser atribuida al hecho de que, durante los últimos años y en un intento de incrementar su cuota de mercado dentro de un entorno de creciente competencia, los bancos más grandes han desarrollado políticas de fijación de precios de sus productos, sobre todo por el lado del activo, más agresivas y caracterizadas por un alto grado de vinculación a los tipos de mercado (prueba de ello es el extraordinario crecimiento de los préstamos indexados). El uso ampliamente extendido de derivados sobre tipos de interés 
por parte de los bancos de mayor dimensión, favorecido por la presencia de economías de escala, puede haber jugado también un papel destacado en este sentido. Todo ello ha desembocado en unas cuentas de resultados y un comportamiento bursátil de los bancos grandes más influenciados por la evolución de las condiciones de mercado. En contraste, la combinación de políticas comerciales menos agresivas, la menor diversificación geográfica y de productos, el uso menos difundido de instrumentos derivados y una trayectoria bursátil ampliamente condicionada por factores de riesgo idiosincrásicos (rumores de posibles operaciones corporativas), pueden estar detrás de la menor vulnerabilidad de los bancos pequeños al riesgo de interés.

Por su parte, el parámetro $\gamma$, asociado a la varianza condicional del rendimiento de las carteras bancarias, ha sido normalmente interpretado como la compensación requerida por los agentes aversos al riesgo por invertir en activos arriesgados. Según esto, un incremento de la volatilidad del rendimiento debería ir acompañado de un mayor rendimiento esperado, con lo cual cabría pensar en un principio que este parámetro debe tomar valores positivos. Sin embargo, dado que el riesgo capturado por la varianza condicional no es el riesgo sistemático no diversificable, sino el riesgo total, el signo del parámetro $\gamma$ no tiene por qué ser necesariamente positivo, ya que en estas circunstancias un aumento del riesgo total no ha de traducirse forzosamente en un rendimiento más elevado. De hecho, si las fluctuaciones de la volatilidad tienen su origen principalmente en shocks de riesgo no sistemático, $\gamma$ podría adoptar cualquier signo ${ }^{(7)}$.

En este caso, los valores estimados de $\gamma$ difieren en términos de signo y magnitud entre las tres carteras bancarias, siendo positivo y significativo para las Carteras G y M, y negativo y significativo para la Cartera P. Esta divergencia podría ser consecuencia de la naturaleza heterogénea de las expectativas de los inversores sobre el comportamiento futuro de las Carteras G, M y P, poniéndose de nuevo de manifiesto el carácter más exógeno de los bancos de menor tamaño. La ausencia de un resultado concluyente con respecto a este parámetro está en línea con la falta de consenso encontrada en la literatura. Así, French et al. (1987) y Campbell y Hentschel (1992) detectaron una vinculación positiva entre riesgo y rendimiento, mientras que Glosten et al. (1993), Elyasiani y Mansur (1998) o Faff et al. (2005) hallaron una relación negativa $(\gamma<0)$. Finalmente, Baillie y DeGennaro (1990) y Ryan y Worthington (2004) obtuvieron un no significativo.

En relación a los parámetros de la ecuación de la varianza condicional, el término constante de dicha ecuación, $\alpha_{0}$, es positivo, significativo y con un valor muy pequeño en todos los casos, lo que implica que el proceso generador de rendimientos de las acciones bancarias tiene un componente de volatilidad invariante en el tiempo de magnitud muy reducida. Los parámetros representativos de los efectos ARCH y GARCH, $\alpha_{1}$ y $\beta$, respectivamente, son positivos y significativos a los niveles usuales para las tres carteras, satisfaciendo el re-

(7) En este sentido, Engle et al. (1987) mostraron que el signo y la magnitud de este parámetro dependen de las funciones de utilidad de los agentes y de las condiciones de oferta de los activos, de forma que su signo no está predeterminado. A su vez, Glosten et al. (1993) sugirieron diversas razones por las que la relación entre riesgo y rendimiento puede llegar a ser negativa. Finalmente, Elyasiani y Mansur (1998) propusieron una explicación alternativa de un coeficiente de aversión relativa al riesgo negativo en el contexto específico del sector financiero. En particular, si los bancos son menos afectados que otros sectores por los shocks económicos entonces los inversores incrementarán sus posiciones en acciones bancarias huyendo de sectores más perjudicados, lo que desembocará en una prima de riesgo más baja para las acciones bancarias. 
quisito de no negatividad y sugiriendo que el comportamiento heteroscedástico del rendimiento de las acciones bancarias puede ser adecuadamente caracterizado por un modelo GARCH(1,1). Para las tres carteras el parámetro $\alpha_{1}$, representativo del impacto del shock ocurrido en el último período, adopta siempre una cuantía sustancialmente más baja que el de la varianza condicional retardada, $\beta$, indicativo del efecto de las sorpresas previas. Este resultado, idéntico al obtenido por Elyasiani y Mansur (1998) y Ryan y Worthington (2004) para otros mercados, implica que el comportamiento bursátil del sector bancario tiene una memoria superior a un período y que la volatilidad es más sensible a sus propios valores retardados que a las nuevas sorpresas en el mercado.

Asimismo, la suma de los parámetros ARCH y GARCH, $\alpha_{1}+\beta$, representativa del grado de persistencia de la volatilidad, es inferior a la unidad para las tres carteras independientemente de la variable de tipo de interés considerada, cumpliendo las condiciones de estacionariedad de segundo orden requeridas por los modelos GARCH. El elevado valor de la medida de persistencia de la volatilidad, cuyos valores oscilan entre 0,81 y 0,93 , muestra que los shocks del sector bancario tienen efectos altamente persistentes y que la función de respuesta de la volatilidad decrece a un ritmo relativamente lento. Estos resultados sugieren que el empleo de un modelo tradicional de volatilidad constante para modelizar el rendimiento de las acciones bancarias del mercado español sería inapropiado.

A su vez, el parámetro $\delta$, que mide el efecto de la volatilidad de los tipos de interés sobre la volatilidad del rendimiento de las carteras bancarias, resulta negativo y significativo para las dos carteras que incorporan la volatilidad de tipos en el modelo estimado (G y M) con independencia de la variable de tipos de interés usada. Además, de forma análoga al resultado obtenido con los movimientos de los tipos de interés, parece existir una relación positiva entre el valor absoluto de este parámetro, el tamaño de las entidades y el plazo hasta el vencimiento del tipo de interés utilizado. Así, la volatilidad de los tipos de interés tiene un impacto negativo más fuerte en términos de magnitud sobre la volatilidad del rendimiento de la cartera cuanto mayor es el tamaño de los bancos integrantes de la cartera y, además, dicha volatilidad incide en mayor medida en el caso de los tipos de interés a largo plazo.

Este resultado indica que ante un aumento de la volatilidad de los tipos de interés la volatilidad del rendimiento de las acciones bancarias tiende a estabilizarse en el siguiente período. Una posible explicación apunta a que en respuesta a un incremento de la volatilidad de tipos los bancos grandes y medianos buscan protegerse del riesgo de interés y son capaces de conseguir, al menos parcialmente, su objetivo en el plazo de un mes mediante, por ejemplo, la contratación de productos derivados o la reducción del gap de duraciones entre activos y pasivos bancarios, de tal forma que se produce una disminución de la volatilidad del rendimiento de sus acciones en el siguiente período mensual.

La consideración conjunta de este último resultado y el concerniente al impacto de los cambios de los tipos de interés pone de relieve que para las Carteras G y M existe un efecto global de los tipos de interés estadísticamente significativo con independencia de la variable de tipos de interés empleada. En consecuencia, puede afirmarse que el sector bancario español presenta una significativa exposición al riesgo de interés, especialmente al utilizar los cambios de los tipos a largo plazo como proxy de las variaciones de los tipos de interés. 
Para comprobar la idoneidad del modelo formulado, se han realizado varios contrastes finales ${ }^{(8)}$. En primer lugar, se ha contrastado si el proceso generador de rendimientos de las carteras bancarias sigue una especificación ARCH $\left(H_{0}: \beta=\delta=\gamma=0\right)$, GARCH $\left(H_{0}: \delta=\gamma=0\right)$, o ARCH-M $\left(H_{0}: \beta=\delta=0\right)$, rechazándose estas hipótesis nulas en todos los casos a un nivel del $1 \%$, lo que parece indicar que la especificación GARCH-M constituye una forma funcional bastante apropiada para modelizar el comportamiento de los rendimientos de las acciones bancarias $^{(9)}$. En segundo lugar, el contraste de signos de Engle y Ng (1993) ha sido aplicado para detectar posibles especificaciones incorrectas relacionadas con la existencia de efectos asimétricos en las carteras bancarias. Los resultados obtenidos sugieren la ausencia de asimetrías, lo que contribuye a reforzar la idoneidad del modelo simétrico planteado.

\subsection{IMPORTANCIA RELATIVA DEL RIESGO DE MERCADO Y DEL RIESGO DE INTERÉS}

Con el objeto de alcanzar una visión más clara de la importancia relativa del riesgo de mercado y del riesgo de interés, se ha realizado un análisis complementario dirigido a determinar la capacidad explicativa individual de cada uno de estos factores sobre el rendimiento de las carteras. Para ello, se ha partido del clásico modelo bifactorial de Stone y, dado que el rendimiento de la cartera de mercado y los cambios de los tipos de interés son independientes debido a la ortogonalización efectuada, la varianza total del rendimiento de una cartera bancaria cualquiera, $\operatorname{Var}\left(R_{i t}\right)$, se puede expresar como:

$$
\operatorname{Var}\left(R_{i t}\right)=\lambda_{2 i} \operatorname{Var}\left(R_{m t}\right)+\theta_{i}^{2} \operatorname{Var}\left(\Delta I_{i t}\right)+\operatorname{Var}\left(\varepsilon_{i t}\right)
$$

Para realizar una comparación adecuada entre ambos factores se ha dividido la ecuación anterior por $\operatorname{Var}\left(R_{i t}\right)$. De esta manera, el porcentaje de contribución de cada factor individual a la varianza total del rendimiento de la cartera vendrá dado por el producto de su correspondiente coeficiente al cuadrado por el cociente entre su varianza y la varianza total del rendimiento de la cartera en cuestión. La Tabla 3 permite constatar que, para las tres carteras bancarias y con independencia del tipo de interés utilizado, los resultados son análogos. Así, se observa que el riesgo de mercado es el determinante más relevante de la variabilidad del rendimiento de cada cartera, mientras que el riesgo de interés tiene una importancia relativa considerablemente inferior, si bien los tipos a largo plazo se revelan nuevamente como los que ejercen una mayor incidencia. Además, se aprecia que la capacidad explicativa de ambos factores disminuye conforme se reduce el tamaño de la cartera, confirmando que las carteras de menor dimensión presentan un comportamiento bursátil más guiado por factores idiosincrásicos.

\subsection{ANÁLISIS POR SUBPERIODOS}

En este epígrafe el modelo GARCH(1,1)-M propuesto en las ecuaciones (1) a (3) ha sido extendido con el propósito de dilucidar si el impacto del riesgo de interés sobre las acciones bancarias se ha mantenido constante a lo largo de todo el período de estudio. Específica-

(8) Los resultados correspondientes a estos contrastes tampoco aparecen por motivos de espacio, aunque pueden ser solicitados a los autores.

(9) En el caso de la Cartera $P$ las hipótesis contrastadas no incluyen, lógicamente, el parámetro $\delta$. 
mente, se ha examinado si la introducción del euro como moneda única en el marco de la Unión Monetaria Europea a partir del 1 de enero de 1999 ha alterado significativamente el grado de exposición al riesgo de interés de las entidades financieras españolas, distinguiendo para ello entre dos subperiodos, esto es, desde enero de 1993 a diciembre de 1998 por un lado y desde enero de 1999 a diciembre de 2005 por otro.

TABLA 3

IMPORTANCIA RELATIVA DE LOS FACTORES DE RIESGO VALORES OBTENIDOS DE $\boldsymbol{R}^{2}$

\begin{tabular}{|c|c|c|c|c|c|c|c|c|c|c|}
\hline & \multicolumn{9}{|c|}{ TIPOS DE INTERÉS } \\
\hline & & \multicolumn{3}{|c|}{3 meses } & \multicolumn{3}{|c|}{10 años } & \multicolumn{3}{|c|}{ Spread } \\
\hline & & $\Delta I_{i, t}$ & $R_{m t}$ & Total & $\Delta l_{i, t}$ & $R_{m t}$ & Total & $\Delta I_{i, t}$ & $R_{m t}$ & Total \\
\hline Cartera G & $\mathrm{R} 2(\%)$ & 0,85 & 53,84 & 54,69 & 2,81 & 51,77 & 54,58 & 1,22 & 53,47 & 54,69 \\
\hline Cartera M & & 1,30 & 34,21 & 35,52 & 2,74 & 32,78 & 35,52 & 1,19 & 34,83 & 36,02 \\
\hline Cartera P & & 1,24 & 15,19 & 16,42 & 5,59 & 12,40 & 17,99 & 1,08 & 15,35 & 16,43 \\
\hline
\end{tabular}

Esta tabla muestra el porcentaje de contribución de los riesgos de mercado y de interés, medidos a través del $R^{2}$ factorial obtenido en base a la expresión (5), a la explicación de la varianza total del rendimiento de cada una de las carteras bancarias.

Dado que el elevado número de parámetros a estimar en los modelos GARCH requiere un considerable tamaño muestral, se ha optado por emplear un procedimiento de variables ficticias en lugar de realizar estimaciones por separado para cada uno de los subperiodos. En este sentido, se ha introducido una variable ficticia, $D$, multiplicando a la serie de cambios de los tipos de interés que toma un valor 1 desde enero de 1993 hasta diciembre de 1998 y 0 en el resto del período muestral. Tal y como ha sido definida esta variable ficticia, su coeficiente asociado, $\eta$, recoge el efecto diferencial en términos de exposición al riesgo de interés que se produce durante el primer subperiodo muestral. Así, la obtención de un parámetro $\eta$ negativo y significativo puede ser interpretada como evidencia de que el efecto del riesgo de interés es sustancialmente más elevado -en valor absoluto- durante el primer subperiodo.

Los resultados de la estimación del modelo extendido con variables ficticias aparecen en la Tabla 4. El parámetro $\eta$ resulta negativo y significativo en la gran mayoría de los casos al emplear los cambios de los tipos a largo y a corto plazo, si bien los resultados no son tan concluyentes al considerar el spread de tipos. Esta evidencia es consistente con la literatura previa (véase, por ejemplo, Akella y Chen, 1990; Kwan, 1991; Faff y Howard, 1999; o Brewer et al., 2007), sugiriendo que la sensibilidad de las acciones bancarias ante los movimientos de los tipos de interés se ha debilitado considerablemente a partir de la entrada en funcionamiento del euro.

Esta reducción del riesgo de interés en el sector bancario durante los últimos años puede ser básicamente atribuida al hecho de que, en respuesta a la mayor volatilidad de las condiciones financieras derivada de la creciente interdependencia entre mercados, las entidades bancarias han adoptado un papel más activo en la gestión de activos y pasivos, desarrollando sistemas más efectivos de medición y gestión del riesgo de interés. Asimismo, el uso recientemente extendido de instrumentos derivados con fines de cobertura también puede haber jugado un papel relevante. 
TABLA 4

EstiMACIÓN DEL MODELo GARCH (1,1)-M EXTENDIDO CON VARIABLE FICTICIA

\begin{tabular}{|c|c|c|c|c|c|c|c|c|c|}
\hline & \multicolumn{3}{|c|}{ Cartera G } & \multicolumn{3}{|c|}{ Cartera $M$} & \multicolumn{3}{|c|}{ Cartera $P$} \\
\hline & 3 meses & 10 años & Spread & 3 meses & 10 años & Spread & 3 meses & 10 años & Spread \\
\hline$\theta$ & $\begin{array}{c}1,94 \\
(0,69)\end{array}$ & $\begin{array}{c}-3,44 \\
(-1,60)\end{array}$ & $\begin{array}{l}-1,88^{\star \star *} \\
(-7,63)\end{array}$ & $\begin{array}{c}2,42 \\
(1,42)\end{array}$ & $\begin{array}{c}2,59 \\
(1,62)\end{array}$ & $\begin{array}{l}-0,73^{\star \star *} \\
(-4,40)\end{array}$ & $\begin{array}{c}-1,69 \\
(-1,59)\end{array}$ & $\begin{array}{c}-0,67 \\
(-0,81)\end{array}$ & $\begin{array}{c}-0,17 \\
(-0,92)\end{array}$ \\
\hline$\eta$ & $\begin{array}{l}-6,52^{* *} \\
(-2,09)\end{array}$ & $\begin{array}{l}-4,69^{\star *} \\
(-1,97)\end{array}$ & $\begin{array}{l}1,52^{\star \star \star} \\
(4,67)\end{array}$ & $\begin{array}{l}-4,57^{\star \star \star} \\
(-4,42)\end{array}$ & $\begin{array}{l}-6,53^{\star * *} \\
(-3,33)\end{array}$ & $\begin{array}{l}0,95^{\star \star *} \\
(5,06)\end{array}$ & $\begin{array}{c}0,13 \\
(0,11)\end{array}$ & $\begin{array}{l}-3,43^{* \star *} \\
(-3,86)\end{array}$ & $\begin{array}{l}-0,61^{\text {** }} \\
(-2,11)\end{array}$ \\
\hline
\end{tabular}

Esta tabla muestra los parámetros estimados del modelo GARCH (1,1)-M univariante extendido que afectan a la serie de variaciones de los tipos de interés. El modelo se ha aplicado a las diferentes carteras bancarias y variables alternativas de tipos de interés consideradas e incluye una variable ficticia, $D$, que permite diferenciar el impacto del riesgo de interés sobre las entidades bancarias en dos subperiodos muestrales (antes y después de la introducción del euro en enero de 1999). El modelo estimado es:

Carteras G y M:

Cartera P:

$$
\begin{gathered}
R_{i t}=\omega_{i}+\lambda R_{i t}+\theta \Delta l_{i t}+\eta_{i t} D \Delta l_{-i t}+\gamma_{i} \log h_{i t}+\varepsilon_{i t} \\
h_{i t}=\alpha_{0}+\alpha_{i} \varepsilon_{i t-1}^{2}+\beta h_{i t-1}+\delta_{i} C V l_{t-1} \\
\varepsilon_{i t} \mid \Omega_{t-1} \sim N\left(0, h_{t}\right)
\end{gathered}
$$

$$
\begin{gathered}
R_{i t}=\omega_{i}+\lambda_{i} R_{m t}+\theta \Delta l_{i t}+\eta_{i} D \Delta \Delta_{-i t}+\gamma_{i} \log h_{i t}+\varepsilon_{i t} \\
h_{i t}=\alpha_{0}+\alpha_{i} \varepsilon_{i t-1}^{2}+\beta h_{i t-1} \\
\varepsilon_{i t} \mid \Omega_{t-1} \sim N\left(0, h_{t}\right)
\end{gathered}
$$

donde $D= \begin{cases}1 & \text { si } t \leq \text { Enero } 1999 \\ 0 & \text { si } t>\text { Enero } 1999\end{cases}$

\section{CONCLUSIONES}

La incidencia del riesgo de interés sobre el valor de las entidades bancarias se ha convertido en una cuestión de indudable trascendencia como resultado de la combinación de factores tales como el sustancial incremento de la volatilidad de los tipos de interés en los últimos años y la nueva regulación sobre requerimientos mínimos de capital para la cobertura de los riesgos bancarios, junto al habitual efecto directo de las variaciones de los tipos de interés sobre los ingresos y costes financieros de las entidades de crédito. En particular, el conocimiento del impacto de los movimientos de los tipos de interés sobre las acciones bancarias resulta de gran relevancia para gestores bancarios, inversores, autoridades supervisoras e incluso académicos de cara al diseño de estrategias de cobertura del riesgo de interés, a las decisiones de asignación de activos o a la evaluación del efecto de las medidas de política monetaria.

En este trabajo se examina la exposición del sector bancario español al riesgo de interés mediante un análisis a nivel de carteras construidas según criterios de tamaño y utilizando diferentes variables alternativas de tipos de interés. A diferencia de la literatura clásica sobre riesgo de interés de las acciones bancarias, centrada básicamente en el impacto de los cambios en el nivel de los tipos de interés, aquí también se presta atención destacada al efecto de la volatilidad de los tipos de interés sobre la distribución de los rendimientos de las carteras bancarias. Con tal fin, una versión extendida de un modelo GARCH-M univariante es empleada como marco de análisis. 
La evidencia empírica obtenida confirma la percepción ampliamente extendida de que el riesgo de interés constituye un importante factor explicativo del proceso generador de rendimientos de las acciones bancarias españolas, si bien es cierto que, como era de prever, desempeña un papel secundario con respecto al riesgo de mercado. En línea con el grueso de la literatura, se pone de relieve que las variaciones de los tipos de interés tienen un efecto negativo sobre el rendimiento de las diferentes carteras bancarias, siendo los tipos a largo plazo los que ejercen una influencia más significativa.

Esta conexión inversa puede ser explicada en base a las siguientes razones. Primero, el planteamiento más común apunta al tradicional desequilibrio de plazos en los balances bancarios, en virtud del cual los activos tienden a presentar un plazo de vencimiento medio superior al de los pasivos, como el principal factor responsable. Segundo, al igual que ocurre con los títulos de renta fija, las acciones bancarias exhiben una correlación negativa con los tipos de interés, estrechamente ligada a su consideración de activos sustitutivos de los bonos. A estos dos argumentos de índole más general, puede añadirse un tercer motivo, vinculado al ciclo económico alcista experimentado por la economía española desde mediados de los años noventa. Específicamente, los resultados del sector bancario español han experimentado un crecimiento espectacular durante los últimos años, con el consiguiente efecto positivo sobre las cotizaciones bursátiles, en un contexto de tipos de interés históricamente bajos. En esencia, se ha tratado de un crecimiento impulsado principalmente por el extraordinario aumento del número de operaciones activas contratadas, sobre todo en el segmento hipotecario, en el marco del boom inmobiliario español.

Además, parece existir una relación directa entre el tamaño de las entidades y su grado de sensibilidad ante los movimientos de los tipos de interés. Esta divergencia podría ser el resultado de diferencias sustanciales entre bancos distinto tamaño en términos de política de fijación de precios de las operaciones bancarias, sobre todo por el lado del activo, del grado de utilización de derivados sobre tipos de interés y otras innovaciones financieras, del nivel de diversificación geográfica y de productos, etc. Así, los bancos de mayor dimensión tienen un comportamiento bursátil ampliamente influenciado por las condiciones de mercado, mientras que los bancos más pequeños son afectados en mayor medida por factores de tipo idiosincrásico.

De manera adicional, se aprecia una conexión significativa entre el riesgo y el rendimiento de las diferentes carteras bancarias, si bien el signo de la misma resulta ambiguo dependiendo de la cartera concreta. En consecuencia, este resultado no contribuye a aclarar la controversia existente en la literatura con respecto a esta cuestión.

Finalmente, la volatilidad de los tipos de interés se configura asimismo como un determinante significativo del comportamiento de las acciones bancarias, constatándose un efecto negativo sobre la volatilidad del rendimiento de las carteras. En este caso también parece haber una relación entre la magnitud en valor absoluto del impacto de la volatilidad de tipos y el tamaño institucional, de tal forma que los bancos más grandes son los que parecen en mejores condiciones de ajustar sus estrategias para protegerse ante un incremento del riesgo de interés asociado a una mayor volatilidad de tipos. 


\section{REFERENCIAS BIBLIOGRÁFICAS}

AкELLA, S. R., y ChEN, S. J., 1990. Interest Rate Sensitivity of Bank Stock Returns: Specification Effects and Structural Changes. Journal of Financial Research 13 (2): 147-154.

Amigo, L., y Rodríguez, F. 2007. Alteraciones en el comportamiento bursátil de las acciones de empresas tecnológicas inducidas por el vencimiento de derivados. Revista Española de Financiación y Contabilidad XXXVI (133): 123-146.

АмоR, B.; TAscón, M. T., y Fanjul, J. L., 2008. Factores determinantes de la rentabilidad anormal de los bancos de la OCDE. Revista Española de Financiación y Contabilidad XXXVII (139): 469-499.

Ballue, R. T., y DeGennaro, R. P. 1990. Stock Returns and Volatility. Journal of Financial and Quantitative Analysis 25 (2): 203-214.

Bartram, S. M. 2002. The Interest Rate Exposure of Nonfinancial Corporations. European Finance Review 6 (1): 101-25.

Bollerslev, T. 1986. Generalized Autoregressive Conditional Heteroscedasticity. Journal of Econometrics 31 (3): 307-327.

Brewer, E.; Carson, J. M.; Elyasian, E.; Mansur, I., y Scott, W.L. 2007. Interest Rate Risk and Equity Values of Life Insurance Companies: A GARCH-M Model. Journal of Risk and Insurance 74 (2): 401423.

Campbell, J. Y., y Hentschel, L. 1992. No News is Good News: An Asymmetric Model of Changing Volatility in Stocks Returns. Journal of Financial Economics 31 (2): 281-318.

CzajA, M.; Scholz, H., and Wilkens, M. 2006. Interest Rate Risk of German Financial Institutions-The Impact of Level, Slope, and Curvature of the Term Structure, Working Paper, Ingolstadt School of Management. Disponible en http://papers.ssrn.com/sol3/papers.cfm?abstract_id=912239 (Consultado el 6 de febrero de 2009).

Deshmukh, S. D.; Greenbaum, S. I., y Kanatas, G., 1983. Interest Rate Uncertainty and the Financial Intermediary's Choise of Exposure. Journal of Finance 38 (1): 141-147.

ELyasiani, E., y Mansur, I. 1998. Sensitivity of the Bank Stock Returns Distribution to Changes in the Level and Volatility of Interest Rate: A GARCH-M Model. Journal of Banking \& Finance 22 (5): 535-563.

ELYASIANI, E., y MansuR, I., 2004. Bank Stock Return Sensitivities to the Long-term and Short-term Interest Rate: A Multivariate GARCH Approach. Managerial Finance 30 (9): 32-45.

EnGLE, R. F. 1982. Autoregressive Conditional Heteroscedasticity with Estimates of the Variance of United Kingdom Inflation. Econometrica 50 (4): 987-1007.

ENGLE, R. F.; LILIEN, D. M., y RoBins, R. P., 1987. Estimating time varying risk premia in the term structure: The ARCH-M model, Econometrica 55 (2): 391-407.

Engle, R. F., y NG, V. K. 1993. Measuring and Testing the Impact of News on Volatility. Journal of Finance 48 (5): 1749-1778.

Faff, R. W.; Hodgson, A., y Kremmer, M. L., 2005. An Investigation of the Impact of Interest Rates and Interest Rate Volatility on Australian Financial Sector Stock Return Distributions. Journal of Business Finance \& Accounting, 32 (5-6): 1001-1032.

FAFF, R. W., y HowARD, P. F. 1999. Interest Rate Risk of Australian Financial Sector Companies in a Period of Regulatory Change. Pacific-Basin Finance Journal 7 (1): 83-101.

FAMA, E. F., y French, K. R, 1993. Common Risk Factors in the Returns on Stocks and Bonds. Journal of Financial Economics, 33 (1): 3-56.

Ferrer, R.; Santomá, J., y Sebastián, A. 1999. El Riesgo de Interés en el Mercado Español de Acciones. Una Aproximación Sectorial. Revista Española de Financiación y Contabilidad XXVIII (98): 43-75. 
FerRer, R.; GonzÁlez, C., y Soto, G. 2008. Análisis Sectorial de la Exposición al Riesgo de Interés de las Empresas Españolas, Información Comercial Española. Revista de Economía, marzo-abril 841: $135-148$.

FlanNery, M. J., y JAMES, C. M. 1984. The Effect of Interest Rate Changes on the Common Stock Returns of Financial Institutions. Journal of Finance 39 (4): 1141-1153.

French, K. R.; Schwert, G. W., y Stambaugh, R. F. 1987. Expected Stock Returns and Volatility. Journal of Financial Economics 19: 3-29.

García Blandón, J. (2008). Rendimientos estacionales en la Bolsa española: importancia del tamaño de la empresa. Revista Española de Financiación y Contabilidad XXXVII (139): 527-540.

Glosten, L. R.; Jagannathan, R., y Runkie, D. 1993. On the Relationship between the Expected Value and the Volatility on the Nominal Excess Returns on Stocks. Journal of Finance 48 (5): 1779-1801.

JaReÑo, F. 2006. Sensibilidad de los Rendimientos Sectoriales a Tipos de Interés Reales e Inflación. Investigaciones Económicas 30 (3): 577-610.

JAREÑO, F. 2008. Spanish stock market sensitivity to real interest and inflation rates: an extension of the Stone two-factor model with factors of the Fama and French three-factor model. Applied Economics 40 (24): 3159-3171.

Joseph, N. L., y Vezos, P., 2006. The Sensitivity of US Banks'Stocks Returns to Interest Rate and Exchange Rate Changes. Managerial Finance 32 (2): 182-199.

Kwan, S. H., 1991. Reexamination of Interest Rate Sensitivity of Commercial Bank Stock Returns Using a Random Coefficient Model. Journal of Financial Services Research 5 (1): 61-76.

Lynge, M. J., y Zumwatt, J. K. 1980. An Empirical Study of the Interest Rate Sensitivity of Commercial Bank Returns: A Multi-Index Approach. Journal of Financial and Quantitative Analysis 15 (3): 731742.

Madura, M., y ZarRuK, E. 1995. Bank exposure to interest rate risk: A global perspective. Journal of Financial Research 18 (1): 1-13.

Poon, S. H., y Granger, C. W. J. 2003. Forecasting Volatility in Financial Markets. A Review. Journal of Economic Literature 41 (2): 478-539.

Ryan, S. K., y Worthington, A. C. 2004. Market, Interest Rate and Foreign Exchange Rate Risk in Australian Banking: A GARCH-M Approach. International Journal of Applied Business and Economic Research 2 (2): 81-103.

Song, F. 1994. A Two factor ARCH Model for Deposit-Institution Stock Returns. Journal of Money, Credit and Banking 26 (2): 323-340.

Stone, B. K. 1974. Systematic Interest Rate Risk in a Two-Index Models of Returns. Journal of Financial and Quantitative Analysis 9: 709-721.

Sweeney, R., y Warga, A. 1986. The Pricing of Interest rate Risk: Evidence from the Stock market. Journal of Finance 41 (2): 393-410.

TAI, C. S., 2000. Time-Varying Market, Interest rate, and Exchange rate Risk Premia in the US Commercial Bank Stock Returns. Journal of Multinational Financial Management 10 (3-4): 397-420.

Yourougou, P. 1990. Interest Rate and the Pricing of Depository Financial Intermediary Common Stock: Empirical Evidence. Journal of Banking and Finance 14 (4): 803-820. 
